\title{
Burnout en estudiantes de pregrado de medicina y su relación con variables de personalidad
}

\author{
María Erika Ortega \\ Godeleva Rosa Ortiz \\ Instituto de Investigaciones Psicológicas, Universidad Veracruzana, México \\ Armando J. Martínez \\ Instituto de Neuroetología y Centro de Ciencias Biomédicas, Universidad Veracruzana, México
}

(Rec: 19 Junio 2014 / Acept: 28 Noviembre 2014)

\begin{abstract}
Resumen
El objetivo de esta investigación fue analizar la asociación entre autoeficacia percibida, personalidad resistente, locus de control, percepción de estrés y síndrome de Burnout en 40 estudiantes de medicina de pregrado. El análisis de los datos se efectuó considerando estadística descriptiva y técnicas exploratorias multivariadas. Los resultados muestran la pertinencia que puede tener la personalidad resistente en la atenuación del estrés crónico al asociarse negativamente con el síndrome de Burnout y agruparse dentro de los componentes que explicaban mayor variación en la combinación lineal de variables en comparación con la autoeficacia y el locus de control. Se confirma la necesidad de reforzar la formación de los estudiantes de medicina a través del establecimiento de recursos de afrontamiento ante el estrés y formación de una personalidad resistente, ya que los datos muestran niveles altos en percepción de estrés y síndrome de Burnout a seis meses de estar realizando el internado.

Palabras clave: personalidad resistente, Burnout, estudiantes de medicina de pregrado y estudio descriptivo.
\end{abstract}

\begin{abstract}
The objective of this research was to analyze the association between perceived self-efficacy, hardiness, locus of control, perceived stress and Burnout syndrome in 40 undergraduate medical students. The data analysis was performed considering descriptive statistics and multivariate exploratory techniques. The results show the relevance that may have the hardiness in the attenuation of chronic stress negatively associated with Burnout and grouped within components that explained more variation in the linear combination of variables compared to self-efficacy and locus of control. The need to strengthen the training of medical students through the establishment of resources Coping Stress and formation of a strong personality is confirmed as the data show high levels of perceived stress and Burnout six months of doing the internship.

KeyWords: hardy personality, Burnout, undergraduate physicians, descriptive study.
\end{abstract}

Correspondencia:

Domicilio: Dr. Luis Castelazo Ayala s/n, Col. Industrial las Ánimas, C.P. 91190. Xalapa, Veracruz, México. Correos electrónicos: orther@hotmail.com; godelevarosa@hotmail.com; armartinez@uv.mx 


\section{Introducción}

La formación de estudiantes de medicina ha sido considerada como una de las carreras donde los alumnos manifiestan mayores niveles de estrés por su alto y creciente nivel de exigencia (Arango, Castaño, Henao, Jiménez, López y Páez, 2010; Brennan, McGrady, Whearty, Lynch, Rapport y Schaefer, 2012; del Toro, Gorguet, Pérez y Ramos, 2011) y por las características de la actividad académica con largas y diversificadas jornadas de aprendizaje (Msaouel et al., 2010), así como por los factores sociales (mayores expectativas y presión social) vinculados a ella (Behere, Yadav y Behere, 2011). El nivel de exigencia y estrés en esta carrera se mantiene desde el inicio al fin e incluso se incrementa según avanzan los estudiantes en su formación, agudizándose más cuando éstos deben realizar su internado de pregrado.

Este internado forma parte del plan de estudios de todas las escuelas y facultades de medicina en México (NOM234-SSA1-2003). Es un requisito obligatorio, previo a la realización del servicio social y al examen profesional (Sánchez, Flores, Urbina y Lara, 2008). Se considera como un periodo fundamental en la formación del estudiante que le permite integrar y consolidar los conocimientos teóricos y prácticos para la atención a la salud de los pacientes (GómezLópez, Rosales-Gracia, Ramírez-Martínez, García-Galaviz, Peña-Maldonado y Vázquez-Vázquez, 2009). Durante un año, los estudiantes deben desarrollar y dominar las competencias correspondientes a las áreas de cirugía, ginecología y obstetricia, medicina familiar, medicina interna, pediatría y urgencias, áreas por las que transitan y donde enfrentan un sinnúmero de situaciones novedosas y demandantes que pueden considerarse como detonantes o agravantes del estrés

Si bien los internos son alumnos del último año de medicina, su compromiso "laboral" es equiparable al de un profesional médico, por lo que sus actividades pueden ser como un "trabajo", en el que deben cumplir horarios y actividades con responsabilidades específicas.

Las actividades que desempeñan durante la atención de los pacientes los exponen, además, al sufrimiento, miedo, angustia o problemas de los pacientes y familiares, e incluso a su muerte. Adicionalmente, los estudiantes carecen de autonomía en sus decisiones, ya que se les considera insuficientemente capacitados (Prins et al., 2010), opinión que se vincula a la incertidumbre personal sobre su propia capacidad y/o conocimientos, así como a la búsqueda constante de alta eficiencia profesional, al considerar que de ellos depende la salud o la vida de una persona (Sánchez et al., 2008).
La jornada laboral que desempeñan puede durar entre 24 y hasta 36 horas continuas, lo que propicia la privación del sueño (Sánchez et al., 2008), la falta de tiempo para el autocuidado, descanso y divertimento (Brennan et al., 2012), con preocupaciones económicas e insuficientes periodos de vacaciones. Estas condiciones ponen a prueba la resistencia, tolerancia, capacidad de respuesta, los conocimientos y el esfuerzo de cada médico interno, inserto en un ambiente hospitalario con altos niveles de estrés, derivados tanto del tipo de actividades que desarrollan, como de la forma que toman las interrelaciones con otros miembros del equipo de salud, usualmente de abuso verbal, subestimación y humillaciones por parte de médicos adscritos, residentes, enfermeras, compañeros de servicio social (Sobowale, Ning, Fan, Liu y Sherer, 2014) y discriminación sexual sobre todo en el caso de las mujeres (Moreno, Villa, Ibarra, Vaquero, Castillo, Alvarado, Nájera y Guevara, 2013).

El alto nivel de estrés que padecen los internos de pregrado sobrepasa su capacidad de afrontamiento y pone en riesgo o alteración su salud psicológica (Schwenk, Davis y Wimsatt, 2010). Diversos estudios han registrado durante este periodo la presencia de trastornos depresivos (Joffre-Velázquez, Martínez-Perales, García-Maldonado y Sánchez-Gutiérrez, 2007; Perales, Sogi y Morales, 2003), trastornos de ansiedad (Bohorquez, 2007), ideas suicidas (Coffin, Álvarez y Marín, 2011; Dyrbye et al., 2008; Sobowale et al., 2014) y abuso de alcohol y drogas (Campos-Castolo, Martínez-López y Chavarría-Islas, 2010). Lo anterior además de la afectación personal, conduce al deterioro académico y a errores en la atención médica con el daño consecuente para los pacientes.

El proceso de deterioro físico y mental de profesionales que trabajan en áreas de la salud ha sido conceptualizado como síndrome de Burnout o desgaste profesional, el cual explica la respuesta emocional de los profesionales expuestos de manera prolongada a estresores relacionados con el esfuerzo para realizar diversas tareas que compiten en tiempos, asumir un sinnúmero de responsabilidades y responder a presiones organizacionales (Maslach, Schaufeli y Leiter, 2001). El síndrome de Burnout (SBO) se integra por tres dimensiones: agotamiento emocional, despersonalización y baja realización personal (Maslach, 2003), que en conjunto suponen desgaste y una problemática interna aguda (Moreno-Jiménez, Garrosa y González, 2000b).

Aunque el origen del SBO se sitúa en condiciones laborales (sobrecarga de trabajo, trato con pacientes difíciles, turnos laborales, etc.), actualmente se insiste en el papel fundamental de las variables de personalidad en la comprensión y explicación del síndrome. Es particularmente el caso de características o recursos de personalidad como 
la autoeficacia percibida, el locus de control y la personalidad resistente, los que pueden actuar como moduladores o amortiguadores del estrés.

La autoeficacia es una variable de cognición específica $\mathrm{y}$ cambiante, que se forma y se reelabora constantemente a partir del procesamiento y la integración de la información procedente de diferentes orígenes: los logros en el pasado, la observación del comportamiento de los demás, la persuasión verbal y la autopercepción del estado fisiológico del organismo. En términos generales, el constructo de autoeficacia representa "la capacidad percibida para ejecutar con éxito un comportamiento específico o en un sentido general, se refiere a la confianza que tenemos sobre nuestra capacidad para enfrentarnos a una gama de situaciones estresantes" (Schwarzer y Hallum, 2008).

El "locus de control" es la convicción de las personas sobre su responsabilidad en sus éxitos y fracasos, así como de las recompensas y castigos que reciben en consecuencia (Laborín, Vera, Durazo y Parra, 2008). En función de donde cree la persona que se ubica la fuente de control, se distinguen 2 factores: el control externo general (control de los eventos a través de la suerte, el destino, la fatalidad, la casualidad, la fortuna y otros aspectos relacionados con el mandato divino) y el locus de control interno (atribución del éxito y/o fracaso debido al propio empeño, esfuerzo, inteligencia o habilidad).

Por último, el constructo de personalidad resistente, o hardiness, representa una característica que influye en la manera en cómo la gente percibe las situaciones y reacciona en circunstancias estresantes. Personas hardiness son más eficaces para hacer frente al estrés y resistencia que protege contra los síntomas de salud inducidos por el estrés (Gerber et al., 2013). Tres dimensiones la integran: compromiso, control y reto que condicionan el tipo de respuesta de la personalidad resistente al estrés. Es decir, más que considerar las nuevas situaciones como una amenaza, se ven como una oportunidad.

En términos generales, las tres variables de personalidad antes descritas han demostrado tener una relación directa con la salud de las personas y un efecto indirecto sobre la salud independientemente del nivel de estrés que se esté experimentando o como amortiguadores de los efectos del mismo.

Con base en lo anterior, se estudió el comportamiento de variables como la personalidad resistente, autoeficacia percibida y el locus de control en médicos internos de pregrado, con el fin de determinar el tipo de relación que guardan y su capacidad predictiva del síndrome de Burnout.

\section{Método}

\section{Participantes y procedimiento}

A partir de tres hospitales públicos de segundo y tercer nivel de atención de la ciudad de Xalapa, Veracruz, México, se integró una muestra de 40 estudiantes de medicina que en 2013 su internado de pregrado. El estudio fue de tipo descriptivo longitudinal (Montero y León, 2007; RamosÁlvarez, Moreno-Fernández, Valdés-Conroy y Catena, 2008). En la primera medición (T1), los participantes respondieron una batería integrada por cinco instrumentos de medición descritos más adelante. Seis meses después, se llevó a cabo la segunda medición (T2) administrando la misma batería de la primera medición y los mismos participantes.

Por razones de temporalidad para el análisis de la información fue necesario formar dos grupos: promoción 2012 (22 estudiantes que al T1 tenían seis meses de estar realizando el internado y al T2 concluían el internado) y promoción 2013 (18 estudiantes que al T1 iniciaban su internado y al T2 cumplían 6 meses en el internado). De los participantes, $60 \%$ eran hombres y $40 \%$ mujeres, con una edad promedio de 23.78 años, $\mathrm{SD}=0.66$ y un rango de 22 a 26 años. El Comité de Bioética en Salud del Estado de Veracruz revisó y aprobó el protocolo de investigación. Todos los estudiantes firmaron el consentimiento informado para la participación en el estudio.

\section{Instrumentos}

Cuestionario general. El instrumento contenía información sociodemográfica como datos laborales, área de rotación, número de horas que pasa en el hospital, número de personas que atiende, etc.

Cuestionario de Desgaste Profesional-Abreviado (CDPE-A) desarrollado por Moreno-Jiménez, Garrosa y González (2000a). Este instrumento evalúa variables asociadas al proceso de desgaste profesional (antecedentes y consecuencias), desgaste profesional (Burnout) y personalidad resistente (hardiness). Tiene 65 ítems ubicados en cinco subescalas con cuatro opciones de respuesta tipo Likert. El cuestionario se ajustó a las características de la población universitaria inmersa en el ambiente hospitalario, en el cual participan los estudiantes de medicina de pregrado. Los valores alfa de Cronbach para las diferentes subescalas del CDPE-A fueron: Escala de Antecedentes $(\alpha=0.72)$, Síndrome Burnout $(\alpha=0.87)$, Escala de Consecuencias $(\alpha=0.92)$ y Escala de Personalidad Resistente $(\alpha=0.86)$. 
Escala de autoeficacia generalizada, desarrollada por Bäßler y Schwarzer (1996). El instrumento evalúa el sentimiento estable de competencia personal para manejar de forma eficaz una gran heterogeneidad de situaciones estresantes. Se utilizó la versión en español con 10 ítems. Su diseño responde a una escala tipo Likert de cuatro puntos, donde 1 significa "incorrecto" y 4 significa "cierto". El puntaje obtenible oscila entre 10 y 40 puntos. El instrumento reporta consistencia interna de $\alpha=0.75$ a $\alpha=0.91$ (Scholz, Gutiérrez, Sud y Schwarzer, 2002) y en este estudio fue de $\alpha=0.87$.

Escala de locus de control. Se utilizó la escala ajustada para Sonora, México por Vera y Cervantes (2000). El instrumento tiene 40 reactivos con siete opciones de respuesta en escala tipo Likert, que van desde "completamente de acuerdo" hasta "completamente en desacuerdo". Estima dos dimensiones de la expresión de control: locus externo, que es la creencia de que son otros elementos y no el esfuerzo propio lo que controla su vida (estos elementos adicionales pueden ser el destino, la suerte, Dios o personas con mayor nivel y poder que uno), y locus interno, que es la percepción de que los éxitos y fracasos son resultado de las propias competencias. La consistencia interna en cada una de las subescalas fue para locus de control externo, $\alpha=$ 0.92, y para locus de control interno: $\alpha=0.95$.

Escala de Percepción de Estrés (PSS). Adaptación para México de González y Landero (2007) de 14 ítems que estiman la intensidad en que las personas se han sentido molestas o preocupadas por algo durante el último mes o si han percibido bienestar o se han sentido seguras de su capacidad para controlar sus problemas personales. La escala, con un diseño tipo Likert de 5 opciones, avanza en un rango de 0 («nunca») a 4 («siempre»). La escala puntúa de 0 a 56 , donde las puntaciones superiores indican un mayor estrés percibido. Se encontró una consistencia interna de $\alpha=0.85$.

\section{Procedimiento}

Se obtuvo la autorización de las autoridades hospitalarias y académicas para realizar la investigación y el consentimiento informado de parte de quienes aceptaron participar de modo que los instrumentos fueron aplicados en los hospitales donde realizan su internado de pregrado.

\section{Análisis estadístico}

Los datos generales fueron analizados mediante estadística descriptiva. Se obtuvieron correlaciones de Pearson para las diferentes variables psicológicas y sus posibles combinaciones. Dentro de las técnicas de ordenación multivariada se recurrió al Análisis de Componentes Principales (ACP), para estimar la responsabilidad de las variables estudiadas sobre la variación observada.

\section{Resultados}

\section{Correlatos promoción 2012}

En la primera medición de las variables de personalidad, la personalidad resistente fue la única que se asoció de forma negativa con las variables SBO, consecuencias y percepción de estrés. Seis meses después (segunda medición) se repite la misma asociación, pero no alcanza el nivel de significancia.

Esta asociación indica que los médicos internos de pregrado con altas puntuaciones en la percepción del estrés

Tabla 1.

Correlaciones para las ocho variables psicológicas medidas en internos de pregrado de medicina (promoción 2012)

\begin{tabular}{|c|c|c|c|c|c|c|c|c|}
\hline Variables Psicológicas & ANT & SBO & CONS & PR & AEG & LCI & LCE & PS \\
\hline$\overline{\mathrm{ANT}}$ & - & 0.61 & 0.55 & 0.09 & -0.18 & 0.03 & -0.12 & 0.42 \\
\hline SBO & 0.29 & -- & 0.65 & -0.19 & -0.50 & -0.14 & 0.07 & 0.57 \\
\hline CONS & 0.21 & 0.66 & -- & -0.29 & -0.27 & 0.06 & 0.11 & 0.24 \\
\hline PR & 0.09 & -0.46 & -0.54 & -- & 0.48 & -0.09 & -0.24 & -0.26 \\
\hline AEG & -0.01 & -0.24 & -0.11 & 0.34 & -- & -0.14 & -0.25 & -0.46 \\
\hline LCI & -0.18 & 0.05 & 0.08 & -0.10 & -0.33 & -- & -0.44 & -0.05 \\
\hline LCE & 0.03 & 0.02 & -0.13 & 0.33 & 0.53 & -0.08 & -- & 0.13 \\
\hline PS & 0.32 & 0.53 & 0.55 & -0.58 & -0.28 & -0.02 & -0.17 & -- \\
\hline
\end{tabular}

Nota: Las correlaciones del pretest se presentan debajo de la diagonal y las correlaciones del postest se presentan arriba de la diagonal. Las correlaciones marcadas en negritas son significativas a $\mathrm{p}<.05000$. $\mathrm{ANT}=$ antecedentes; $\mathrm{SBO}=$ síndrome de $\mathrm{Burnout}$; $\mathrm{CONS}=$ consecuencias; $\mathrm{PR}=$ personalidad resistente; $\mathrm{AEG}=$ autoeficacia; $\mathrm{LCI}=$ locus de control interno; $\mathrm{LCE}=$ locus de control externo; $\mathrm{PS}=$ percepción de estrés. 
Tabla 2.

Análisis de componentes principales para el grupo de internos de pregrado (promoción 2012)

\begin{tabular}{ccccc}
\hline & Antes & \multicolumn{2}{c}{ Después } \\
& PC 1 & PC 1 & PC 2 & PC 3 \\
\hline ANT & 0.27 & -0.65 & -0.51 & -0.34 \\
SBO & 0.76 & $\mathbf{- 0 . 8 7}$ & -0.13 & -0.21 \\
CONS & 0.78 & $\mathbf{- 0 . 7 3}$ & -0.20 & -0.08 \\
PR & -0.78 & 0.45 & -0.43 & -0.58 \\
AEG & -0.51 & 0.69 & -0.25 & -0.42 \\
LCI & 0.16 & 0.02 & -0.54 & $\mathbf{0 . 7 4}$ \\
LCE & -0.38 & -0.23 & $\mathbf{0 . 8 1}$ & -0.22 \\
PS & 0.80 & $\mathbf{0 . 7 1}$ & 0.03 & -0.01 \\
Expl. Var & 2.97 & 2.97 & 1.54 & 1.30 \\
\% Var total & 0.37 & 0.37 & 0.19 & 0.16 \\
\hline
\end{tabular}

Nota: Números en negritas indican alta carga factorial. $\mathrm{ANT}=$ antecedentes; $\mathrm{SBO}=$ síndrome de Burnout; $\mathrm{CONS}=$ consecuencias; $\mathrm{PR}=$ personalidad resistente; $\mathrm{AEG}=$ autoeficacia; $\mathrm{LCI}=$ locus de control interno; $\mathrm{LCE}=$ locus de control externo; $\mathrm{PS}=$ percepción de estrés.

Tabla 3.

Correlaciones para las ocho variables psicológicas medidas en internos de pregrado de medicina (promoción 2013)

\begin{tabular}{|c|c|c|c|c|c|c|c|c|}
\hline $\begin{array}{c}\text { Variables } \\
\text { Psicológicas }\end{array}$ & ANT & SBO & CONS & PR & AEG & LCI & LCE & PS \\
\hline ANT & -- & 0.77 & -0.56 & -0.57 & -0.24 & -0.25 & 0.10 & 0.55 \\
\hline SBO & 0.86 & -- & -0.57 & -0.59 & -0.24 & -0.20 & 0.12 & 0.79 \\
\hline CONS & -0.69 & -0.83 & -- & 1.00 & 0.34 & 0.22 & -0.29 & -0.43 \\
\hline PR & -0.73 & -0.84 & 0.99 & -- & 0.36 & 0.23 & -0.30 & -0.44 \\
\hline AEG & -0.09 & -0.23 & 0.40 & 0.39 & -- & -0.10 & 0.13 & -0.39 \\
\hline LCI & -0.07 & -0.08 & -0.27 & -0.24 & -0.14 & -- & -0.89 & -0.29 \\
\hline LCE & -0.14 & 0.09 & 0.04 & 0.03 & -0.28 & -0.31 & -- & 0.28 \\
\hline PS & 0.68 & 0.82 & -0.76 & -0.77 & -0.17 & 0.14 & -0.05 & -- \\
\hline
\end{tabular}

Nota. Las correlaciones del pretest se presentan debajo de la diagonal y las correlaciones del postest se presentan arriba de la diagonal. Las correlaciones marcadas en negritas son significativas a $\mathrm{p}<.05000$. ANT = antecedentes; $\mathrm{SBO}=$ síndrome de $\mathrm{Burnout}$; $\mathrm{CONS}=$ consecuencias; $\mathrm{PR}=$ personalidad resistente; $\mathrm{AEG}=$ autoeficacia; $\mathrm{LCI}=$ locus de control interno; $\mathrm{LCE}=$ locus de control externo; $\mathrm{PS}=$ percepción de estrés

muestran bajas puntuaciones en los indicadores de personalidad resistente.

\section{Componentes principales (promoción 2012)}

Los puntajes de los diferentes instrumentos variaron en la ordenación multivariada con el ACP. La combinación lineal de las variables registradas para los estudiantes de pregrado promoción 2012, a los seis meses de su internado, solo explicó el $37 \%$ de la variación en el componente principal 1 (CP1), que se integró por las variables consecuencias del estrés, $\mathrm{SBO}$, personalidad resistente y percepción de estrés.
Al final del internado, el CP1 explicaba el $37 \%$ de la variabilidad, a través de las variables SBO, consecuencias y percepción de estrés. El CP2, integrado por locus externo, explicó el 19\%. Y por último en el CP3 el locus de control interno explicó el 16\% de la variación.

\section{Correlatos promoción 2013}

En la primera medición (inicio de internado), la variable personalidad resistente se asoció significativamente de forma negativa con las variables antecedentes, SBO y percepción de estrés, acorde con lo esperado. Las variables autoeficacia 
Tabla 4.

Análisis de Componentes Principales para el grupo de internos de pregrado (promoción 2013)

\begin{tabular}{|c|c|c|c|c|c|c|}
\hline & \multicolumn{3}{|c|}{ Antes } & \multicolumn{3}{|c|}{ Después } \\
\hline & PC 1 & PC 2 & PC 3 & PC 1 & PC 2 & $\mathrm{PC} 4$ \\
\hline ANT & 0.83 & -0.00 & 0.38 & -0.78 & -0.16 & -0.26 \\
\hline SBO & 0.93 & 0.18 & 0.21 & -0.83 & -0.19 & -0.17 \\
\hline CONS & -0.95 & 0.07 & 0.15 & 0.84 & 0.09 & 0.17 \\
\hline PR & -0.96 & 0.04 & 0.12 & 0.85 & 0.09 & 0.15 \\
\hline AEG & -0.36 & -0.33 & 0.74 & 0.40 & 0.49 & -0.71 \\
\hline LCI & 0.16 & -0.69 & -0.59 & 0.43 & -0.82 & -0.15 \\
\hline LCE & -0.02 & 0.86 & -0.24 & -0.41 & 0.85 & 0.16 \\
\hline PS & 0.86 & -0.05 & 0.12 & -0.77 & -0.06 & 0.26 \\
\hline Expl. Var. & 4.31 & 1.39 & 1.21 & 3.91 & 1.75 & 0.78 \\
\hline$\%$ Var. total & 0.53 & 0.17 & 0.15 & 0.48 & 0.21 & 0.09 \\
\hline
\end{tabular}

Nota: Números en negritas indican alta carga factorial. $\mathrm{ANT}=$ antecedentes; $\mathrm{SBO}=$ síndrome de Burnout; $\mathrm{CONS}=$ consecuencias; $\mathrm{PR}=$ personalidad resistente; $\mathrm{AEG}=$ autoeficacia; $\mathrm{LCI}=$ locus de control interno; $\mathrm{LCE}=$ locus de control externo; $\mathrm{PS}=$ percepción de estrés.

y locus de control interno, aun cuando se asociaron en la dirección esperada, los valores no resultaron significativos. Seis meses después, la variable personalidad resistente se asoció de manera negativa con antecedentes y SBO, esto es, a mayor puntuación en personalidad resistente, menor puntuación en antecedentes y síndrome de Burnout.

\section{Componentes principales (promoción 2013)}

Los médicos de pregrado de la promoción 2013 explican el 54\% de la variación a través del CP1 integrado por antecedentes, SBO, consecuencias, personalidad resistente y percepción de estrés. El CP2 explica el 17\% a través del locus de control externo y la autoeficacia en el CP3 lo hace para el $15 \%$ de la variación.

A los seis meses de estar realizando su internado, el CP1 explicó el $49 \%$ de la variación nuevamente con las variables antecedentes, SBO, consecuencias, personalidad resistente y percepción de estrés. En cambio, el CP2 explicó el 22\% y la contribución fue de los indicadores locus interno y externo.

\section{Discusión}

Los estudiantes de medicina de pregrado están expuestos a altos niveles de estrés desde el inicio de la licenciatura y su clímax llega durante el internado de pregrado en los hospitales, debido a la doble condición que asume el interno, como estudiante aún en formación y como personal no asalariado en un ambiente hospitalario que le asigna responsabilidades y exigencias específicas.
Los resultados encontrados no pueden ser generalizables a otras poblaciones por provenir de una muestra con limitaciones espaciales y temporales, sin embargo, proporcionan elementos importantes a la comprensión del desgaste profesional, al confirmar algunas de las relaciones teóricas esperadas entre el SBO y la personalidad resistente. El estudio muestra cómo las condiciones hospitalarias de enseñanza afectan la estabilidad psicológica de los internos durante su formación profesional al hallar que aproximadamente el $70 \%$ de los estudiantes mostraron niveles altos de síndrome de Burnout y alta percepción de estrés, dato similar al de Guille, Clark, Amstadter y Sen (2014), quienes reportaron que al $81 \%$ de los participantes de su estudio, clasificados como "sensibles al estrés", se les asoció con un probable diagnóstico de depresión durante al menos una evaluación de seguimiento durante su año de internado. Dato que se corrobora al comparar los niveles de estrés y Burnout cuando ambos grupos tienen seis meses de estar realizando su internado, y se encuentra un incremento de aproximadamente $5 \%$ en ambos grupos comparado con la otra medición que se tiene de ambos grupos. Lo anterior refuerza la idea de incorporar a los planes de estudio contenidos para formar recursos de afrontamiento al estrés en los estudiantes de medicina, como una medida protectora para su salud mental y para la prevención de la presencia del Burnout o desgaste profesional.

Al hallar mayor consistencia de asociación en las tres muestras analizadas entre la variable personalidad resistente con las variables antecedentes, consecuencias, percepción de estrés, como con el SBO, se confirma lo reportado en 
diversos estudios (Dolbiert, Smith y Steinhardt, 2007; Judkins, Massey y Huff, 2006; Garrosa, Rainho, Moreno-Jiménez y João, 2010; Moreno, Morett, Rodríguez y Morante, 2006), que la ha ubicado como una variable de personalidad con capacidad moderadora o efecto protector en relación con el nivel de estrés y Burnout. Es decir, se muestra el efecto positivo que la personalidad resistente puede tener en la prevención del proceso de Burnout. Esto se apoya con la combinación lineal de los componentes principales, donde se muestra la estrecha correspondencia entre el SBO y la personalidad resistente.

En resumen, este estudio muestra la pertinencia que tiene la personalidad resistente en la atenuación y manejo del estrés crónico, lo que puede ser determinante en la prevención del SBO y destaca la necesidad de formar recursos de afrontamiento en los estudiantes de medicina. Para confirmar nuestros resultados se sugiere, en una segunda etapa, medir las variables con muestras más grandes y en diversos espacios geográficos, así como formular, ante las instancias correspondientes, las propuestas para incorporar en el plan de estudios la capacitación de los alumnos en los recursos de afrontamiento que caracterizan a este tipo de personalidad.

\section{Referencias}

Arango, A.S., Castaño, C.J.J., Henao, R.C.J., Jiménez, A.D.P., López, H.A.F. y Páez, C.M.L. (2010). Síndrome de Burnout y factores asociados en estudiantes de I a X semestre de la Facultad de Medicina de la Universidad de Manizales (Colombia), 2009. Archivos de Medicina, $10,110-126$.

Bäßler, J. y Schwarzer, R. (1996). Evaluación de la autoeficacia: Adaptación española de la escala de autoeficacia general. Ansiedad y Estrés, $2,1-8$.

Behere, S.P., Yadav, R. y Behere, P.B. (2011). A Comparative Study of Stress Among Students of Medicine, Engineering, and Nursing. Indian Journal Psychology Medicine, 33, 145-148.

Brennan, J., McGrady, A., Whearty, K., Lynch, D., Rapport, D. y Schaefer, P. (2012). Emotionalk status of third year medical students and their responses to a brief intervention. Annals of Behavioral Sciences and Medical Education, 18, 10-14.

Campos-Castolo, E.M., Martínez-López, J. y Chavarría-Islas, R. (2010). Encuesta exploratoria sobre seguridad de los pacientes en médicos internos de pregrado. Revista CONAMED, 15, 116-124.

Coffin, C.N., Álvarez, Z.M. y Marín, C.A. (2011). Depresión e ideación suicida en estudiantes de la FESI: Un estudio piloto. Revista Electrónica de Psicología de Iztacala, 14, 341-354.

Dolbiert, C.L., Smith, S.E. y Steinhardt, M.A. (2007). Relationships of protective factors to stress and symptoms of Illness. American Journal Health Behavior, 31, 423-433.

Dyrbye, L.N., Thomas, M.R., Massie, F.S., Power, D.V., Eacker, A., Harper, W., Durning, S., Moutier, C., Szydlo, D.W., Novotny, P.J., Sloan, J.A. y Shanafelt, T.D. (2008). Burnout and suicidal ideation among US medical students. Annals of Internal Medicine, 149, 334-41.

Garrosa, E., Rainho, C., Moreno-Jiménez, B. y João, M.M. (2010). The relationship between job stressors, hardy personality, coping resources and Burnout in a sample of nurses: A correlational study at two time points. International Journal Nursing Studies, 42, 205-215.

Gerber, M., Kalak, N., Lemola, S., Clough, P.J., Perry, J.L., Pühse, U., Elliot, C., Holsboer-Trachsler, E. y Brand, S. (2013). Are adolescents with high mental toughness levels more resilient against stress? Stress and Health, 29, 164-171.

Gómez-López, V., Rosales-Gracia, S., Ramírez-Martínez, J., GarcíaGalaviz, J., Peña-Maldonado, A. y Vázquez-Vázquez, A. (2009). Evaluación del impacto del internado de pregrado en la solución de problemas clínicos. Gaceta Médica Mexicana, 145, 501-504

González, R.M.T. y Landero, H.R. (2007). Factor structure of the Perceived Stress Scale (PSS) in a simple from México. Spanish Journal of Psychology, 10, 199-206.

Guille, C., Clark, S., Amstadter, A.B. y Sen, S. (2014). Trajectories of depressive symptoms in response to prolonged stress in medical interns. Acta Psychiatrica Scandinavia, 129, 109-115.

Judkins, S., Massey, C. y Huff, B. (2006). Hardiness, stress, and use of ill-time among nurse managers: is there a connection? Nursing Economic, 24, 187-192.

Laborín, A.J.F., Vera, N.J.A., Durazo, S.F.F. y Parra, A.E.M. (2008). Composición del locus de control en dos ciudades latinoamericanas. Revista Psicología desde el Caribe, 22, 63-83.

Maslach, C., Schaufeli, W.B. y Leiter, M.P. (2001). Job Burnout. Annual Review Psychology, 52, 397-422.

Maslach, C. (2003). Job Burnout: New Directions in Research and Intervention. Current Directions in Psychological Science, 12, 189-192.

Msaouel, P., Keramaris, N.C., Tasoulis, A., Kolokythas, D., Syrmos, N., Pararas, N., Thireos, E. y Lionis, C. (2010). RBeusearrnchout and training satisfaction of medical residents in Greece: will the European Work Time Directive make a difference? Human Resources for Health, 8, 1-11.

Montero, I., y León, O.G. (2007). A guide for naming research studies in Psychology. International Journal of Clinical and Health Psychology, 7, 847-862. Recuperado el 7 de noviembre de 2014 de http://www.aepc. es/ijchp/GNEIP07_es.pdf

Moreno, J.B., Garrosa, H.E. y González, G.J.L. (2000a). El desgaste profesional de enfermería. Desarrollo y validación factorial del CDPE. Archivos de Prevención de Riesgos Laborales, 3, 18-28.

Moreno, J.B., Garrosa, H.E. y González, G.J.L. (2000b). Personalidad resistente, Burnout y salud. Escritos de Psicología, 4, 64-77.

Moreno, J.B., Morett, N.N.I., Rodríguez, M.A. y Morante, B.M.E. (2006). La personalidad resistente como variable moduladora del síndrome de Burnout en una muestra de bomberos. Psicothema, 18, 413-418.

Moreno, T.L.M.A., Villa, R.A.R., Ibarra, A.N., Vaquero, E.E., Castillo, S.M., Alvarado, L.N.Y., Nájera, N.L.V. y Guevara, R.E.S. (2013). ¿Eres virgen? -No necesito ser virgen para estar en cirugía. La situación de las médicas internas de pregrado frente a la violencia de género. En: Pacheco, L. de G.L., Cayeros, L.L.I., González, R.F., Murillo, B.A., Navarro, H.M del R., Pont, S.E.S. y Sandoval, A.D. (Eds.). La equidad de género en el desorden de la violencia. Nuevos y viejos escenarios (pp 71-80). México: Universidad Autónoma de Nayarit.

Prins, J.T., Hoekstra-Weebers, J.E.H.M., Gazendam-Donofrio, S.M., Dillingh, G.S., Bakker, A.B., Huisman, M., Jacobs, B. y van der Heijden, F.M.M.A. (2010). Burnout and engagement amog resident doctor in the Netherlands: a national study. Medical Education, 44, 236-247.

Ramos-Álvarez, M.M., Moreno-Fernández, M.M., Valdés-Conroy, B., y Catena, A. (2008). Criteria of the peer-rewiev process for publication of experimental and quasiexperimental research Psychology: A guide for creating researchs papers. International Journal of Clinical and Health Psychology, 8, 751-764. Recuperado el 7 de noviembre de 2014, de http://www.aepc.es/ijchp/ramos-es.pdf.

del Toro, A.A.Y., Gorguet, P.M., Pérez, I.Y. y Ramos, G.D.A. (2011). Estrés académico en estudiantes de medicina de primer año con bajo rendimiento escolar. MEDISAN, 15(1), 17-22. Recuperado el 18 de septiembre de 2014, de http://bvs.sld.cu/revistas/san/vol_15_1_11/ san03111.pdf.

Sánchez, F.A., Flores, E.R., Urbina, B.R. y Lara, F.N.L. (2008). Expectativas y realidades del internado médico de pregrado. Un estudio cualitativo. Investigación en Salud, X, 14-21. 
Scholz, U., Gutiérrez-Doña, B., Sud, S. y Schwarzer, R. (2002). Is general self-efficacy a universal construct? Psychometric findings from 25 countries. European Journal Psychological Assessment, 18, 242-251.

Schwarzer, R. y Hallum, S. (2008). Perceived teacher self-efficacy as a predictor of job stress and Burnout: Mediation Analyses. Applied Psychology: An International Review, 57, 152-171.

Schwenk, T.L., Davis, L. y Wimsatt, L.A. (2010). Depression, Stigma, and Suicidal Ideation in medical Students. Journal of the American Medical Association, 304, 1181-1190.
Sobowale, K., Ning, Z.A., Fan, J., Liu, N. y Sherer, R. (2014). Depression and suicidal ideation in Medical students in China: a call for wellness curricula. International Journal of Medical Education, 5, 31-36.

Vera, J.A. y Cervantes, N.E. (2000). Locus de control en el norte de México. Psicología y Salud, 10, 237-247. 